\title{
Exploring a nonperturbative method for calculation of the anomalous magnetic moment of the electron
}

\section{Sophia CHABYSHEVA*}

University of Minnesota-Duluth, USA

and Southern Methodist University, USA

E-mail: sophia@physics.smu.edu

The electron's anomalous magnetic moment is computed in a light-cone Hamiltonian approach, with Pauli-Villars (PV) particles as UV regulators. The eigenstate is obtained by diagonalizing a matrix that represents the discretization of 48 coupled integral equations for the one-photon/oneelectron wave functions. This generalizes earlier work on a one-photon truncation and extends the eigenstate expansion to two photons. In addition to the physical particles, one PV electron flavor and two PV photon flavors are included in the basis. The second PV photon allows the solution to have a very smooth, slowly varying dependence on the PV electron mass. This in turn allows the numerical approximation to use smaller mass ratios, which reduces round-off errors. An intermediate calculation that retains the one-photon contribution and the two-photon selfenergy contributions yields a much improved result. Where the older, one-photon calculations of the anomalous moment by S.J. Brodsky et al. differed by $14 \%$ from the correct value, inclusion of the self- energy contribution reduces the discrepancy to less than $2 \%$.

LIGHT CONE 2008 Relativistic Nuclear and Particle Physics

July 7-11, 2008

Mulhouse, France

* Speaker. 


\section{Introduction}

The anomalous magnetic moment of the electron is predicted by perturbative QED to very high precision as [1] $a_{e} \equiv(g-2) / 2=\frac{\alpha}{2 \pi}-0.328478965\left(\frac{\alpha}{\pi}\right)^{2}+1.17611\left(\frac{\alpha}{\pi}\right)^{3}-1.434\left(\frac{\alpha}{\pi}\right)^{4}$. This is in excellent agreement with experiment and provides an important check for nonperturbative methods. Here we describe progress toward such a check for a light-cone Hamiltonian method that uses Pauli-Villars (PV) particles as the ultraviolet regulators. The electron eigenstate is obtained within a truncated basis, and the anomalous moment is computed from the wave functions. The one-photon truncation has been solved analytically [2]. The new work involves inclusion of twophoton states, as well as additional analysis of the one-photon truncation at finite PV masses.

The regularization is done by including enough PV particles in the Lagrangian. The formulation is then covariant and, at least for QED, gauge invariant. These symmetries are then broken by the truncation of the Fock-state expansion, but the effect of the breaking is expected to be small.

The existence of a meaningful Fock-state expansion, with well defined wave functions as the coefficients, is made possible by the choice of light-cone quantization [3]. The vacuum is then simple and the projection of the lower eigenstates onto the higher Fock sectors can be small. This is not the case for equal-time quantization [ [

Usually in QED, light-cone gauge is chosen, to simplify constraints; however, in this case, three PV electrons were found to be not enough to properly regulate the theory [2]. There was a strong dependence on PV masses and a lack of equivalence between light-cone perturbation theory and standard covariant perturbation theory. To work in this gauge requires an additional PV photon and higher-order derivative counterterms [2].

In Feynman gauge, one PV electron and one PV photon are in principle sufficient at our level of truncation. The PV interactions can be arranged to cancel the usual instantaneous electron interactions and to simplify the fermionic constraint equations. This worked well for very large PV electron masses [2]. However, if the infinite PV-electron-mass limit is not approached, there remains some strong dependence on the PV photon mass, which can be eliminated by including a second PV photon. This is important for the two-photon truncation, which must be handled numerically and is vulnerable to large round-off errors in the quadratures when the PV electron mass is very large.

The renormalization is handled by requiring the mass $M$ of the lowest eigenstate in the singleelectron sector to be the physical mass of the electron, $m_{e}$. This fixes the bare-mass parameter $m_{0}$ of the Lagrangian as a function of the PV masses. The coupling $\alpha$ is unrenormalized in our truncation, because there are no antifermion contributions. In practice, the eigenvalue problem is solved at fixed $M=m_{e}$ with $\alpha$ obtained from the eigenvalue, and $m_{0}$ is determined by requiring $\alpha$ to take the correct physical value.

This approach differs from that of sector-dependent renormalization [5, 6], where the bare parameters are given different values in each Fock sector. In [6] this has yielded wave functions that are not well defined. The probability of the lowest Fock sector becomes negative if the PV masses are too large, and the expectation value for the number of physical photons is infinite for any choice of PV masses. These difficulties are overcome to some extent by only computing quantities with respect to some external probe and separately renormalizing the external coupling. 
The limitation to finite PV masses occurs also in our approach, due to uncancelled divergences [凤], but the wave functions always yield probabilities between zero and one.

\section{Feynman-gauge light-cone QED}

The Lagrangian of PV-regulated QED in Feynman gauge is

$$
L=\sum_{i=0}^{2}-\frac{1}{4}(-1)^{i} F_{i}^{\mu v} F_{i, \mu \nu}-\frac{1}{2} \sum_{i=0}^{2}(-1)^{i}\left(\partial^{\mu} A_{i \mu}\right)^{2}+\sum_{i=0}^{1}(-1)^{i} \bar{\psi}_{i}\left(i \gamma^{\mu} \partial_{\mu}-m_{i}\right) \psi_{i}-e \bar{\psi} \gamma^{\mu} \psi A_{\mu}
$$

where

$$
A_{\mu}=\sum_{i=0}^{2} \sqrt{\xi_{i}} A_{i \mu}, \quad \psi=\sum_{i=0}^{1} \psi_{i}, \quad F_{i \mu \nu}=\partial_{\mu} A_{i v}-\partial_{v} A_{i \mu} .
$$

The subscript $i=0$ denotes a physical field and $i=1$ or 2 a PV field. The constants $\xi_{i}$ satisfy the following constraints:

$$
\sum_{i=0}^{2}(-1)^{i} \xi_{i}=0, \sum_{i=0}^{2}(-1)^{i} \xi_{i} \mu_{i}^{2} \ln \frac{\mu_{i}}{m_{1}}=0,
$$

with $\xi_{0}=1$. The first constraint guarantees that $A_{\mu}$ is a zero-norm field. The second arranges the cancellation of the leading dependence on the PV electron mass, $m_{1}$.

The coupling of the two zero-norm fields $A_{\mu}$ and $\psi$ as the interaction term reduces the fermionic constraint equation to a solvable equation without forcing the gauge field $A^{+}$to zero. For the null combination $\psi_{0}+\psi_{1}$ that couples to $A^{+}$the constraint on the nondynamical components is the same as the constraint for a free fermion.

The regularization scheme does have the disadvantage of breaking gauge invariance, through the presence of "flavor" changing currents where a physical fermion can be transformed to a PV fermion or vice versa. However, the breaking effects disappear in the limit of large PV fermion mass [2], because the physical fermion cannot make a transition to a state with infinite mass.

Without antifermion terms, the result for the Hamiltonian is [2]

$$
\begin{aligned}
P^{-} & =\sum_{i, s} \int d \underline{p} \frac{m_{i}^{2}+p_{\perp}^{2}}{p^{+}}(-1)^{i} b_{i, s}^{\dagger}(\underline{p}) b_{i, s}(\underline{p})+\sum_{l, \mu} \int d \underline{k} \frac{\mu_{l}^{2}+k_{\perp}^{2}}{k^{+}}(-1)^{l} \varepsilon^{\mu} a_{l \mu}^{\dagger}(\underline{k}) a_{l \mu}(\underline{k}) \\
& +\sum_{i, j, l, s, \mu} \int d \underline{p} d \underline{q}\left\{b_{i, s}^{\dagger}(\underline{p})\left[b_{j, s}(\underline{q}) V_{i j, 2 s}^{\mu}(\underline{p}, \underline{q})+b_{j,-s}(\underline{q}) U_{i j,-2 s}^{\mu}(\underline{p}, \underline{q})\right] \sqrt{\xi_{l}} a_{l \mu}^{\dagger}(\underline{q}-\underline{p})+\text { h.c. }\right\},
\end{aligned}
$$

where the vertex functions are given in [2]. We work in the frame where the total $\vec{P}_{\perp}$ is zero. The eigenstate with total $J_{z}= \pm \frac{1}{2}$ then has the following Fock-state expansion:

$$
\begin{aligned}
& \left|\psi^{ \pm}(\underline{P})\right\rangle=\sum_{i} z_{i} b_{i \pm}^{\dagger}(\underline{P})|0\rangle+\sum_{i j s \mu} \int d \underline{k} C_{i j s}^{\mu \pm}(\underline{k}) b_{i s}^{\dagger}(\underline{P}-\underline{k}) a_{j \mu}^{\dagger}(\underline{k})|0\rangle \\
& \quad+\sum_{i j k s \mu v} \int d \underline{k_{1}} d \underline{k_{2}} C_{i j k s}^{\mu v \pm}\left(\underline{k_{1}}, \underline{k_{2}}\right) \frac{1}{\sqrt{1+\delta_{j k} \delta_{\mu v}}} b_{i s}^{\dagger}\left(\underline{P}-\underline{k_{1}}-\underline{k_{2}}\right) a_{j \mu}^{\dagger}\left(\underline{k_{1}}\right) a_{k v}^{\dagger}\left(\underline{k_{2}}\right)|0\rangle+\ldots,
\end{aligned}
$$

The wave functions $C_{i j s}^{\mu \pm}$ are by determined the mass eigenvalue problem $P^{+} P^{-}|P\rangle=M^{2}|P\rangle$.

As it stands, the eigenstate can lead to unphysical answers, because the PV contributions have negative norm. We extract a physical state by means of a projection onto the physical subspace [7]. 
The projection is accomplished by expressing Fock states in terms of positively normed creation operators $a_{0 \mu}^{\dagger}, a_{2 \mu}^{\dagger}$, and $b_{0 s}^{\dagger}$ and the null combinations $a_{\mu}^{\dagger}=\sum_{i} \sqrt{\xi_{i}} a_{i \mu}^{\dagger}$ and $b_{s}^{\dagger}=b_{0 s}^{\dagger}+b_{1 s}^{\dagger}$. The $b_{s}^{\dagger}$ particles are annihilated by the generalized electromagnetic current $\bar{\psi} \gamma^{\mu} \psi$; thus, $b_{s}^{\dagger}$ and $a_{\mu}^{\dagger}$ create unphysical contributions that are dropped. The anomalous moment is computed only after making this projection.

\section{Solution of the eigenvalue problem}

In the one-photon truncation, the wave functions satisfy the coupled integral equations

$$
\left[M^{2}-m_{i}^{2}\right] z_{i}=\int d \underline{k} \sum_{j, l, \mu} \sqrt{\xi_{l}}(-1)^{j+l} \varepsilon^{\mu}\left[P^{+} V_{j i+}^{\mu *}(\underline{k}, \underline{P}) C_{j l+}^{\mu+}(\underline{k})+P^{+} U_{j i+}^{\mu *}(\underline{k}, \underline{P}) C_{j l-}^{\mu+}(\underline{k})\right]
$$

and

$$
\begin{aligned}
& {\left[M^{2}-\frac{m_{i}^{2}+k_{\perp}^{2}}{(1-y)}-\frac{\mu_{l}^{2}+k_{\perp}^{2}}{y}\right] C_{i l \pm}^{\mu \pm}(\underline{k})=\sqrt{\xi_{l}} \sum_{j}(-1)^{j} z_{j} P^{+} V_{i j \pm}^{\mu}(\underline{k}, \underline{P}),} \\
& {\left[M^{2}-\frac{m_{i}^{2}+k_{\perp}^{2}}{(1-y)}-\frac{\mu_{l}^{2}+k_{\perp}^{2}}{y}\right] C_{i l \mp}^{\mu \pm}(\underline{k})=\sqrt{\xi_{l}} \sum_{j}(-1)^{j} z_{j} P^{+} U_{i j \pm}^{\mu}(\underline{k}, \underline{P}) .}
\end{aligned}
$$

The wave functions $C_{i l \pm}^{\mu \pm}$ are found explicitly in terms of the bare amplitudes $z_{i}$, by inverting the second set of equations, and then eliminated from the first set, to obtain, with use of the definitions of the vertex functions [纤,

$$
\begin{aligned}
&\left(M^{2}-m_{i}^{2}\right) z_{i}=2 e^{2} \sum_{j}(-1)^{j}\left[M^{2} z_{j} \bar{J}+m_{i} z_{j} m_{j} \bar{I}_{0}\right. \\
&\left.-2 M z_{j}\left(m_{i}+m_{j}\right) \bar{I}_{1}\right] .
\end{aligned}
$$

Here

$$
\begin{aligned}
\bar{I}_{n} & =\int \frac{d y d k_{\perp}^{2}}{16 \pi^{2}} \sum_{j l} \frac{(-1)^{j+l} \xi_{l}}{M^{2}-\frac{m_{j}^{2}+k_{\perp}^{2}}{1-y}-\frac{\mu_{l}^{2}+k_{\perp}^{2}}{y}} \frac{\left(m_{j} / M\right)^{n}}{y(1-y)^{n}}, \\
\bar{J} & =\int \frac{d y d k_{\perp}^{2}}{16 \pi^{2}} \sum_{j l} \frac{(-1)^{j+l} \xi_{l}}{M^{2}-\frac{m_{j}^{2}+k_{\perp}^{2}}{1-y}-\frac{\mu_{l}^{2}+k_{\perp}^{2}}{y}} \frac{\left(m_{j}^{2}+k_{\perp}^{2}\right) / M^{2}}{y(1-y)^{2}} .
\end{aligned}
$$

The form of Eq. (3.4) matches that of the equivalent eigenvalue problem in Yukawa theory [7, with $M$ used as the mass scale instead of $\mu_{0}$ and with the replacements $g^{2} \rightarrow 2 e^{2}$ and $I_{1} \rightarrow-2 \bar{I}_{1}$. Consequently, the solution for the one-photon truncated eigenvalue problem is

$$
\alpha_{ \pm}=\frac{\left(M \pm m_{0}\right)\left(M \pm m_{1}\right)}{8 \pi M\left(m_{1}-m_{0}\right)\left(2 \bar{I}_{1} \pm \bar{I}_{0}\right)}, z_{1}=\frac{M \pm m_{0}}{M \pm m_{1}} z_{0}
$$

with $z_{0}$ determined by normalization.

The plot in Fig. 1 shows $\alpha_{ \pm} / \alpha$ as functions of $m_{0}$. The $\alpha_{-}$branch is the physical choice, because the no-interaction limit $\left(\alpha_{-}=0\right)$ corresponds to the bare mass $m_{0}$ becoming equal to the physical electron mass. If the PV electron has a sufficiently large mass, the value of $m_{0}$ that yields 


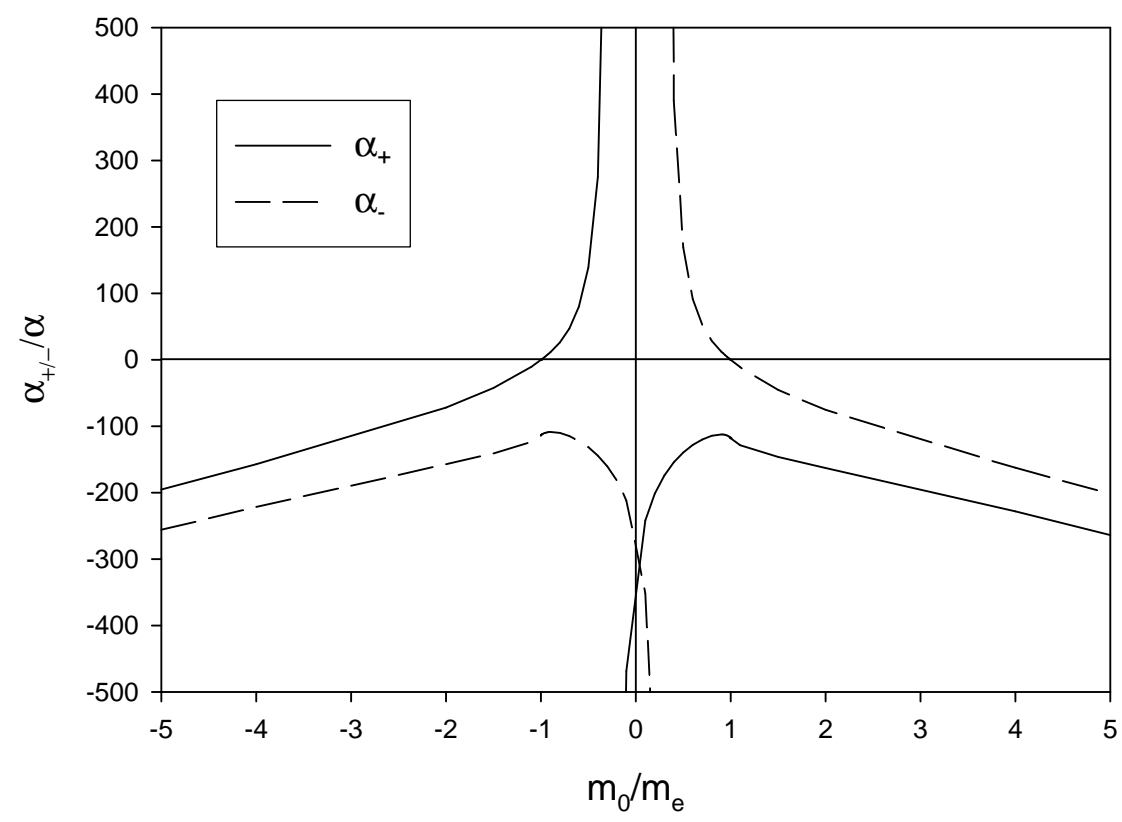

Figure 1: The two solutions of the one-photon eigenvalue problem, for PV mases $m_{1}=1000 m_{e}, \mu_{1}=10 m_{e}$, and $\mu_{2}=\infty$. The horizontal line shows where $\alpha_{ \pm}=\alpha$. The $\alpha_{-}$branch corresponds to the physical choice, but with $m_{0}$ less than $m_{e}$.

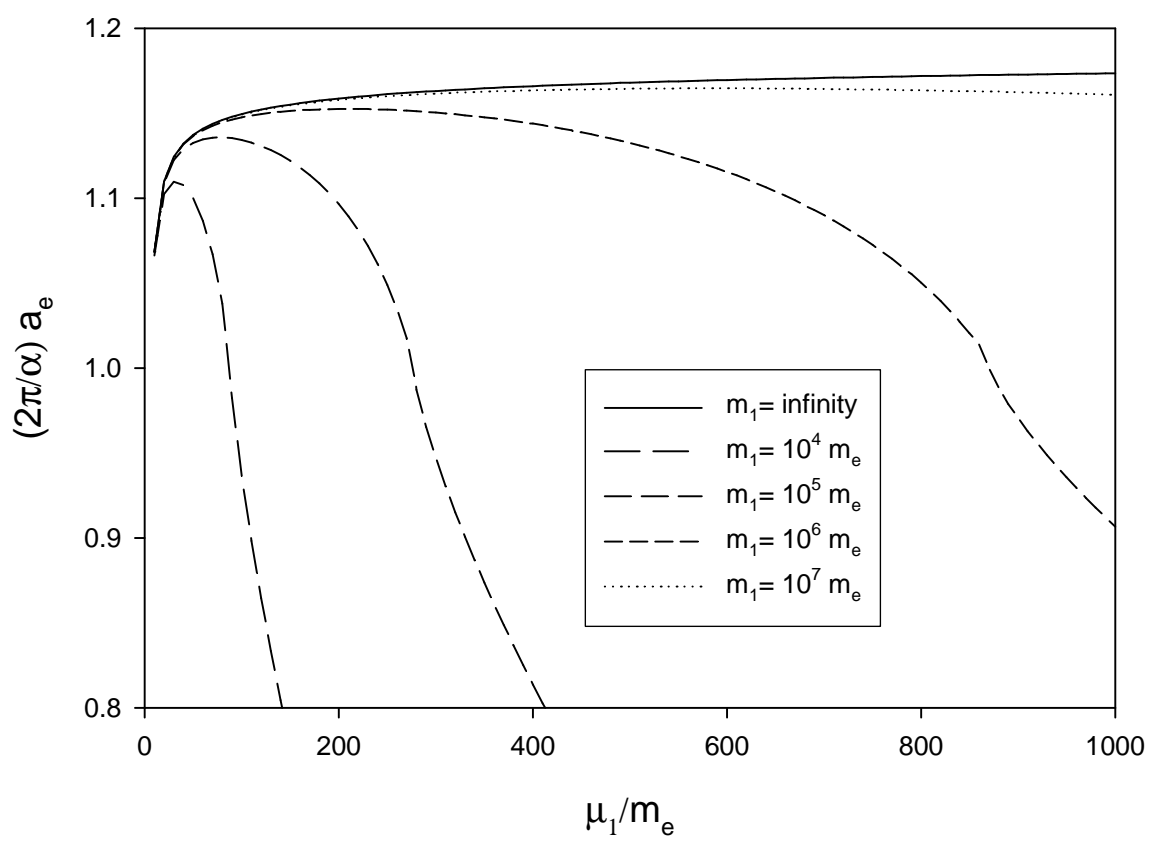

Figure 2: The anomalous moment of the electron in units of the Schwinger term $\left(\frac{\alpha}{2 \pi}\right)$ plotted versus the PV photon mass, $\mu_{1}$, for a few values of the PV electron mass, $m_{1}$, with the second PV photon mass, $\mu_{2}$, set to infinity. 
$\alpha_{-}=\alpha$ is less than $m_{e}$. In this case, the integrals $\tilde{I}_{n}$ and $\tilde{J}$ contain poles for $j=l=0$ and are defined by a principal-value prescription [2].

When the second PV photon is removed via the $\mu_{2} \rightarrow \infty$ limit, the results for the anomalous moment have a very strong dependence on the PV electron mass $m_{1}$, as can be seen in Fig. 2. This strong dependence arises from the sensitivity of the anomalous moment integral to the masses of the constituents, in particular the bare electron mass $m_{0}$. The leading finite- $m_{1}$ correction to the bare electron mass is of the form $\frac{\mu_{1}^{2} \ln \left(\mu_{1} / m_{1}\right)}{8 \pi^{2} m_{e} m_{1}}$. This requires $m_{1}$ to be much larger than $\mu_{1}^{2} / m_{e}$. Such behavior comes from the contribution of $\tilde{I}_{1}$ to the relationship between $m_{0}$ and $\alpha$ in (3.7). Relative to the value $\tilde{I}_{1}(\infty)$ at infinite $m_{1}$, we have

$$
\tilde{I}_{1} \simeq \tilde{I}_{1}(\infty)+\sum_{j=1}^{2} \xi_{j}(-1)^{j} \frac{\mu_{j}^{2} \ln \left(\mu_{j} / m_{1}\right)}{8 \pi^{2} m_{e} m_{1}} .
$$

The second constraint (2.3) on the coupling factors $\xi_{j}$ reduces this leading correction to zero.

In the two-photon truncation, we can eliminate the three-body wave function and the barefermion amplitudes to obtain 48 coupled equations for the two-body wave functions

$$
\begin{aligned}
- & \frac{2 \pi}{\alpha} f_{i j s}^{\mu \pm}\left(y, q_{\perp}\right)=\frac{I_{i j, 1-i}\left(y, q_{\perp}\right)}{1-y} \eta_{1-i, j}\left(y, q_{\perp}\right) f_{1-i, j s}^{\mu \pm}\left(y, q_{\perp}\right) \\
& +\sum_{i^{\prime} j^{\prime} s^{\prime} \mu^{\prime}} \varepsilon^{\mu^{\prime}} \int_{0}^{1} d y^{\prime} d q_{\perp}^{\prime 2} J_{i j s, i^{\prime} j^{\prime} s^{\prime}}^{(0) \mu \mu^{\prime}}\left(y, q_{\perp} ; y^{\prime}, q_{\perp}^{\prime}\right) \eta_{i^{\prime} j^{\prime}}\left(y^{\prime}, q_{\perp}^{\prime}\right) f_{i^{\prime} j^{\prime} s^{\prime}}^{\mu^{\prime} \pm}\left(y^{\prime}, q_{\perp}^{\prime}\right) \\
& +\sum_{i^{\prime} j^{\prime} s^{\prime} \mu^{\prime}} \varepsilon^{\mu^{\prime}} \int_{0}^{1-y} d y^{\prime} d q_{\perp}^{\prime 2} J_{i j s, i^{\prime} j^{\prime} s^{\prime}}^{(2) \mu \mu^{\prime}}\left(y, q_{\perp} ; y^{\prime}, q_{\perp}^{\prime}\right) \eta_{i^{\prime} j^{\prime}}\left(y^{\prime}, q_{\perp}^{\prime}\right) f_{i^{\prime} j^{\prime} s^{\prime}}^{\mu^{\prime} \pm}\left(y^{\prime}, q_{\perp}^{\prime}\right),
\end{aligned}
$$

where $I_{i j k}$ is the self-energy contribution and $J_{i j s, i^{\prime} j^{\prime} s^{\prime}}^{(n) \mu \mu^{\prime}}$ is the kernel with $n$ intermediate photons. The diagonal self-energy contribution is included in the redefined wave functions

$$
f_{i j s}^{\mu \pm}\left(y, q_{\perp}=C_{i j s}^{\mu \pm}\left(y, q_{\perp}\right) /\left(M^{2} \eta_{i j}\left(y, q_{\perp}\right)\right)\right.
$$

with

$$
\eta_{i j}\left(y, q_{\perp}\right)=\frac{y(1-y)}{q_{\perp}^{2}+y\left(m_{i}^{2}+I_{i j i}\left(y, q_{\perp}\right)+(1-y) \mu_{j}^{2}-y(1-y) M^{2}\right.} .
$$

The full solution of this eigenvalue problem is in progress. As an intermediate step, we can solve it with only the two-photon self-energy corrections, that is, with $J^{(2)}$ set to zero. The solution is analytic up to the evaluation of certain integrals, which can be done numerically with some care and effort, especially with respect to poles, to an accuracy of order $1 \%$. The anomalous moment computed from this solution is shown in Fig. 3. along with the one-photon-truncation results. We see that, although the one-photon result differs by $\sim 14 \%$ [2], our new result, the filled circles of Fig. 3, is consistent with perturbative QED, showing only the variations expected from the numerical errors in calculating the integrals.

\section{Acknowledgments}

This work was supported in part by the US Department of Energy and the Minnesota Supercomputing Institute. 


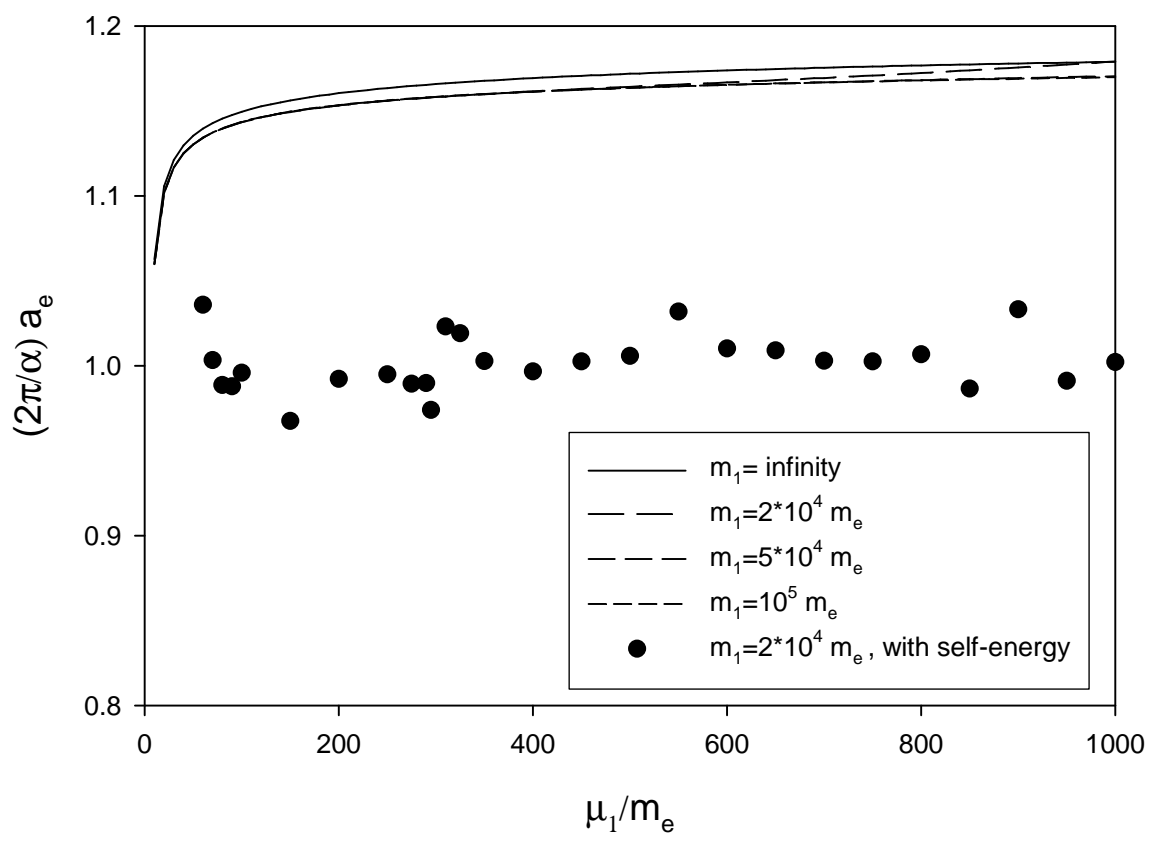

Figure 3: Same as Fig. 2 but with $\mu_{2}=\sqrt{2} \mu_{1}$ and with inclusion of the effect of keeping the two-photon self-energy corrections.

\section{References}

[1] M. Maggiore, A Modern Introduction to Quantum Field Theory, Oxford University Press, Oxford 2005.

[2] S.J. Brodsky, V.A. Franke, J.R. Hiller, G. McCartor, S.A. Paston, and E.V. Prokhvatilov, A nonperturbative calculation of the electron's magnetic moment, Nucl. Phys. B 703, 333 (2004).

[3] P.A.M. Dirac, Forms of relativistic dynamics, Rev. Mod. Phys. 21, 392 (1949).

[4] S.J. Brodsky, H.-C. Pauli, and S.S. Pinsky, Quantum chromodynamics and other field theories on the light cone, Phys. Rep. 301, 299 (1998).

[5] R.J. Perry, A. Harindranath, and K.G. Wilson, Light-front Tamm-Dancoff field theory, Phys. Rev. Lett. 65, 2959 (1990); R.J. Perry and A. Harindranath, Renormalization in the light-front Tamm-Dancoff approach to field theory, Phys. Rev. D 43, 4051 (1991); J.R. Hiller and S.J. Brodsky, Nonperturbative renormalization and the electron's anomalous moment in large- $\alpha Q E D$, Phys. Rev. D 59, 016006 (1998).

[6] V.A. Karmanov, J.-F. Mathiot, and A.V. Smirnov, Systematic renormalization scheme in light-front dynamics with Fock space truncation, Phys. Rev. D 77, 085028 (2008).

[7] S.J. Brodsky, J.R. Hiller, and G. McCartor, The mass renormalization of nonperturbative light-front Hamiltonian theory: An illustration using truncated, Pauli-Villars-regulated Yukawa interactions, Ann. Phys. 305, 266 (2003). 